Journal of Innovative Image Processing (JIIP)

(2020)

Vol.02/ No. 01

Pages: 55-64

https://www.irojournals.com/iroiip/

DOI:_https://doi.org/10.36548/jiip.2020.1.006

\title{
Quantization with Perception for Performance Improvement in HEVC for HDR Content
}

\author{
Mr. H. James Deva Koresh, \\ Research Scholar, \\ Karunya University, \\ Coimbatore, India. \\ Email id: jamesdeva@karunya.edu.in
}

\begin{abstract}
Human eye always reveals a non -linear understanding, for the disturbances caused by the lossy image and video coding. This is mainly because of the masking capability of the human eye to conceal the attributes such as contrast, luminous, spatial and temporal frequencies. To have a distortion less and efficient video encoding for the high dynamic video range content by eluding the invisible messages in the video that causes disturbances the paper puts forth the quantization with perception utilizing the luminous masking. The methodology utilized, computes the tone mapping to scale every frames in the HDR and later quantizes on unit basis with perception tuning. For this purpose the mechanism put forth incorporates the reference model of the HEVC with the extension range of the HEVC. The proposed model was validated by evaluating the reduction incurred in each rate of bit compared to the HDR range extension. The results acquired proved to have an enhancement in terms of the savings endured in the bit rate compared to the High efficient video coding that relied on the high dynamic range visible difference predictor-II
\end{abstract}

Keywords: High Efficient Video Coding, High Dynamic Range Compression, Quantization with Perception, Invisible Information's, Tone Mapping

\section{Introduction}

The traditional devices that were utilized in capturing and as well as displaying, engaged the twenty four bit format called the low standard dynamic range for encoding the pixel, and was capable of only covering a dynamic range and color scale up to a certain limit. The level of luminosity and the range of the colors in the real world varies drastically when captured by the imaging based on the standard or low dynamic range. The human eye has the capacity to adapt to a wide range of luminous levels that varies from the $10^{-5}-10 \mathrm{~cd} / \mathrm{m}^{2}$ and $10-10^{6} \mathrm{~cd} / \mathrm{m}^{2}$ this is known as the scotopic and photopic conditions respectively.

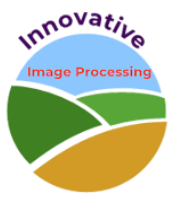


Journal of Innovative Image Processing (JIIP)

(2020)

Vol.02/ No. 01

Pages: 55-64

https://www.irojournals.com/iroiip/

DOI:_https://doi.org/10.36548/jiip.2020.1.006

The HDR technology for imaging enables to have an even higher degree of involvement that are similar to or even better compared to the human eye. The visual benefit of HDR content is greatest when an HDR display shows content. Innovative display technologies uses ground light sources (pad of LED's with the capability of being regulated to improve the contrast of the content) that could be spatially dimmed to adjust LCD section's brightness and magnify its color spectrum and dynamic range. The high dynamic ranging uses the floating point values to represent the original scenarios degrees of luminance and the range of colors. This immersion enhancement is achieved in the HDR by increasing the depth and the rate of the bit which in turn increases the bandwidth and as well as the memory requirement for the video or the images in HDR limiting its adoption. So this paves for even more efficient compression techniques as a solution.

Some of the formats used in the HDR imaging such as the RGBE, open EXR, Tiff, etc. employ the lossless compression for preserving the values of the pixels in the visible color as well as the dynamic range an some employs the integer values for representing the pixel elements of the image. One of the highly challenging work in the process of compression is to retain the quality of the image. For this purpose the compression in the existing method was categorized as the back ward and the perception. The "backwardcompatible HDR compression" methods retain compatibility with legacy 8-bit decoders to decode and view HDR at ease tone-mapped on bequest displays and the "Perception-based compression" of HDR rely on the use of human eye frame work to capture the different masking effects encountered by human eye under HDR conditions accurately. This is done to elude the wastage of bits caused due to the repeated invisible messages in the image or video.

The experiment outcomes of the luminance masking followed in the previous methods showed that the application of the "perceptual uniform transformation" makes the human eye insensitive to the coding disturbances caused in the regions of the HDR images with increased or decreased luminance. For this a masking is done by coding the original image as integer values that are related to the bit depth defined. Utilizing the values the average intensity of integer values associated with the bit depth is connected to the coded lumps to elude the invisible portions that cause disturbance.

So the paper put forth the masking of luminous to preserve the quality of the image employing the computation of tone mapping curve for scaling each frames of a HDR and following the quantization by perceptual tuning to enhance the performance of the HEVC for the HDR content. This methodology put forward is arranged with the related works in 2, proposed work in three, and the performance improvement analysis in 4 and the conclusion in 5. 
Journal of Innovative Image Processing (JIIP)

(2020)

Vol.02/ No. 01

Pages: 55-64

https://www.irojournals.com/iroiip/

DOI:_https://doi.org/10.36548/jiip.2020.1.006

\section{Related Works}

The section presents the works with the few compression strategies followed previously in the high dynamic range. In the methodology put forth by the author Yang et al [1] the compression was divide as the backward and tone mapping and used in coding the images or the video as shown in the figure. 1 below

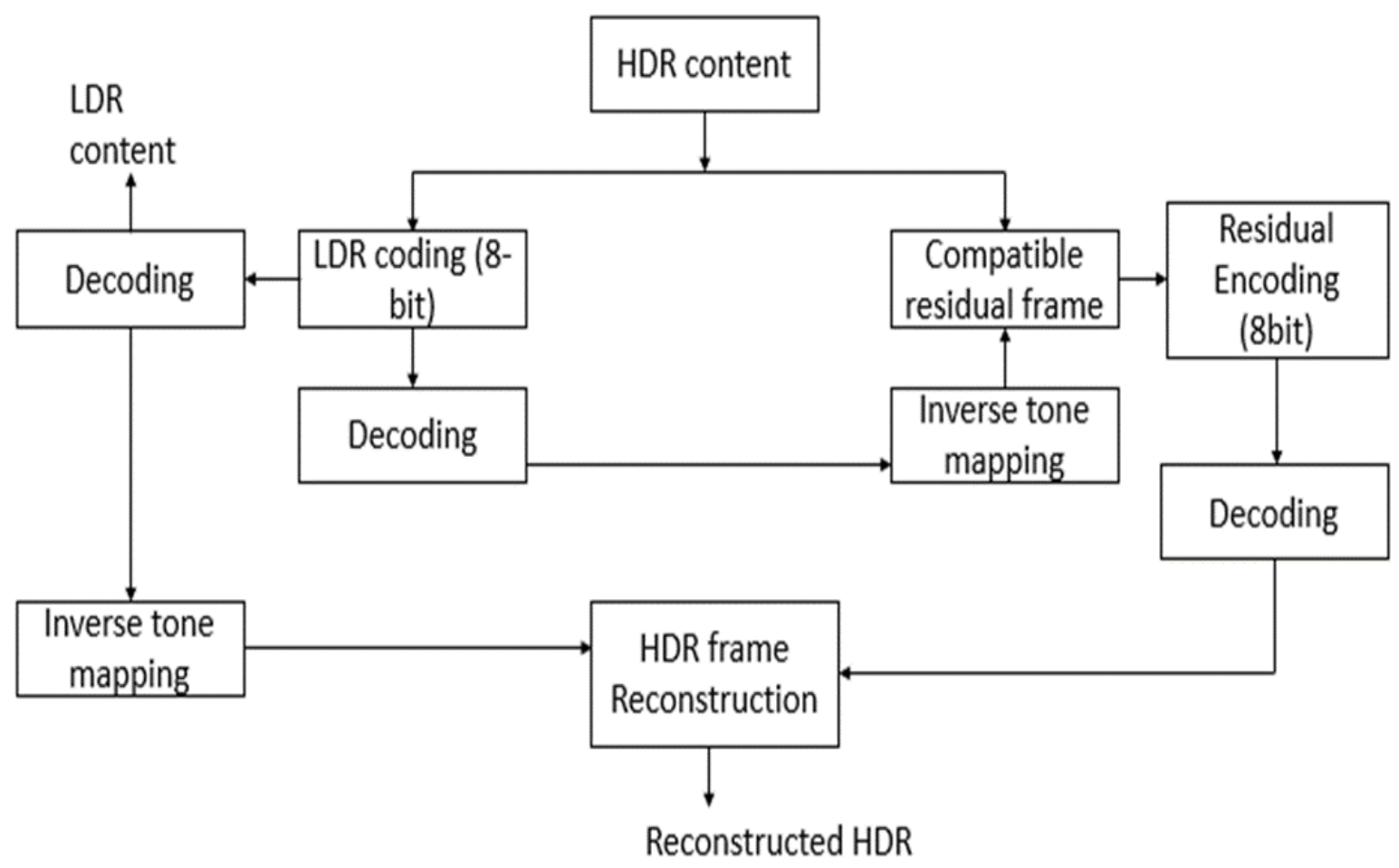

Figure .1 HDR Codecs

Whereas the author Korshunov et al [2] put forward the valuation strategies to decide among the various tone mapping procedures available. More over the author Miller et al [3] in his paper conduct the quantizing process using the perception to enhance the quality of the images in high dynamic range with the depth of bits ranging from 10 to 12. Further the perceptual conversion utilizing the Lloyd Max quantizer to indicate the fourteen bit luminance channel was proposed by the Erik Reinhard et al [4] the visible distortion degree was structured based on its human eye sensitivity to disturbance by the author Wu et al [5]

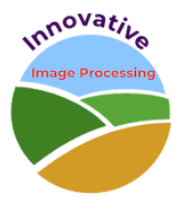


Journal of Innovative Image Processing (JIIP)

(2020)

Vol.02/ No. 01

Pages: 55-64

https://www.irojournals.com/iroiip/

DOI:_https://doi.org/10.36548/jiip.2020.1.006

Further the author M. Mrak, et al [6] in his paper put forth quantization method based on the coarser to quantize the brighter as well as the darker sections of the image utilizing the nonlinear mapping. Kumar, T. Senthil et al [7] put forth the innovative procedure to compress the video retaining its quality using the Chroma subsampling and the luminance masking. Manoharan, Samuel et al [8] devised the "A smart image processing algorithm for text recognition, information extraction and vocalization for the visually challenged." Suma, V. et al [9] conducted the "review on Computer Vision for Human-Machine Interaction"

Koresh, M. H., and J. Deva et al [10] performed the "Computer vision based traffic sign sensing for smart transport." Shakya, Subarna et al [11] put forth an. "Virtual Restoration of Damaged Archeological Artifacts Obtained from Expeditions Using 3D Visualization." Chandy, Abraham et al [12] devised the "RGBD Analysis for Finding the Different Stages of Maturity of Fruits in Farming." Bindhu, V et al [13]. Performed the "Biomedical Image Analysis Using Semantic Segmentation."

\section{Proposed Work}

Masking strategy followed by the human eye is utilized to enhance the efficiency of compression in the High dynamic range. The perception based quantization is followed taking into consideration the attributes of the human eye that is capable of adapting to spatial-temporal nature of the coded video. The perception based quantization utilized, does the masking based on intensity and the content adaption. The entire intensity masking of the human eye disturbance sensitivity is a curve that is U-shaped, this describes the maximum tolerable disturbance degree for the total intensity value. The content adaption is assured by the computing the average intensity for each and every block on which is the quantization is done during the process of encoding. The intensity value defined enables us to have the maximum amount of distortion, that is tolerable for the given intensity value. The figure. 2 presents the block diagram of the proposed compression. 
Journal of Innovative Image Processing (JIIP)

(2020)

Vol.02/ No. 01

Pages: 55-64

https://www.irojournals.com/iroiip/

DOI:_https://doi.org/10.36548/jiip.2020.1.006

Encoder

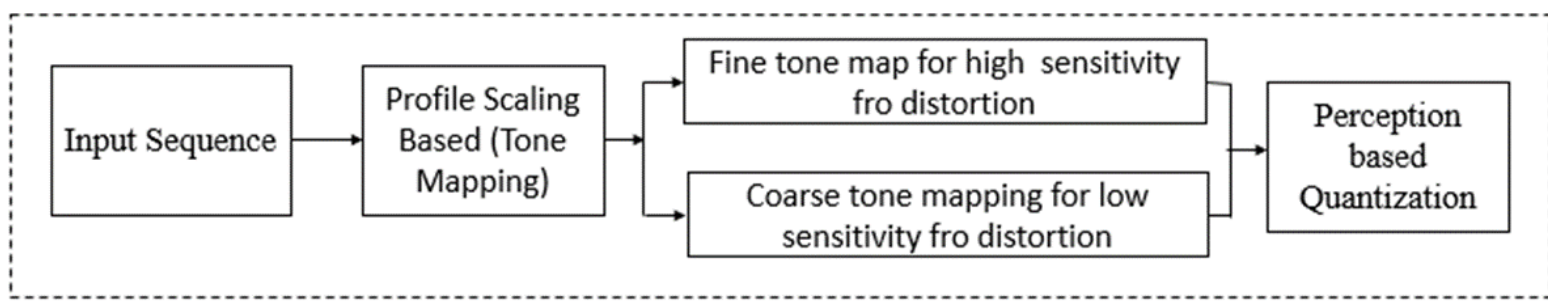

Decoder

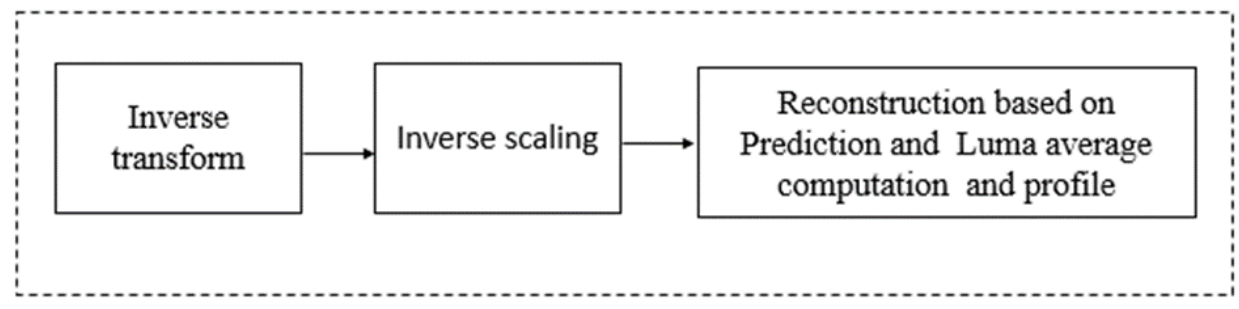

Figure.2 Block Diagram of Proposed

A connection in each step of quantizing is maintained by taking into consideration the error of the quantization $\left(Q_{e r r}\right)$ which is given as shown in the, equation 1

$$
Q_{e r r}=F q_{B l k}-F q_{b l k \prime}
$$

Where the $F q_{B l k}$ and the $F q_{b l k}$, represents the frequency co-efficient of the particular, particular block and the reconstructed coefficient respectively. Further the proposed method utilizes the nonlinear tone mapping to improve the quality of the image minimizing the high variations in the brightness and have a proper content adaptability. The tone mapping conserves the look of the image by maintain the features such as the fixation points of the visual attention and the contrast in the intensity. The proposed approach utilizes the finer mapping of tone for high sensitiveness to distortion and uses the tone mapping that is coarse for the lesser sensitiveness to distortion.

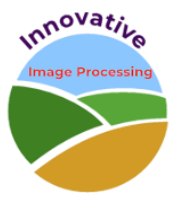


Journal of Innovative Image Processing (JIIP)

(2020)

Vol.02/ No. 01

Pages: 55-64

https://www.irojournals.com/iroiip/

DOI:_https://doi.org/10.36548/jiip.2020.1.006

The flow chart below in the figure. 3 shows the procedures of the proposed methodology.

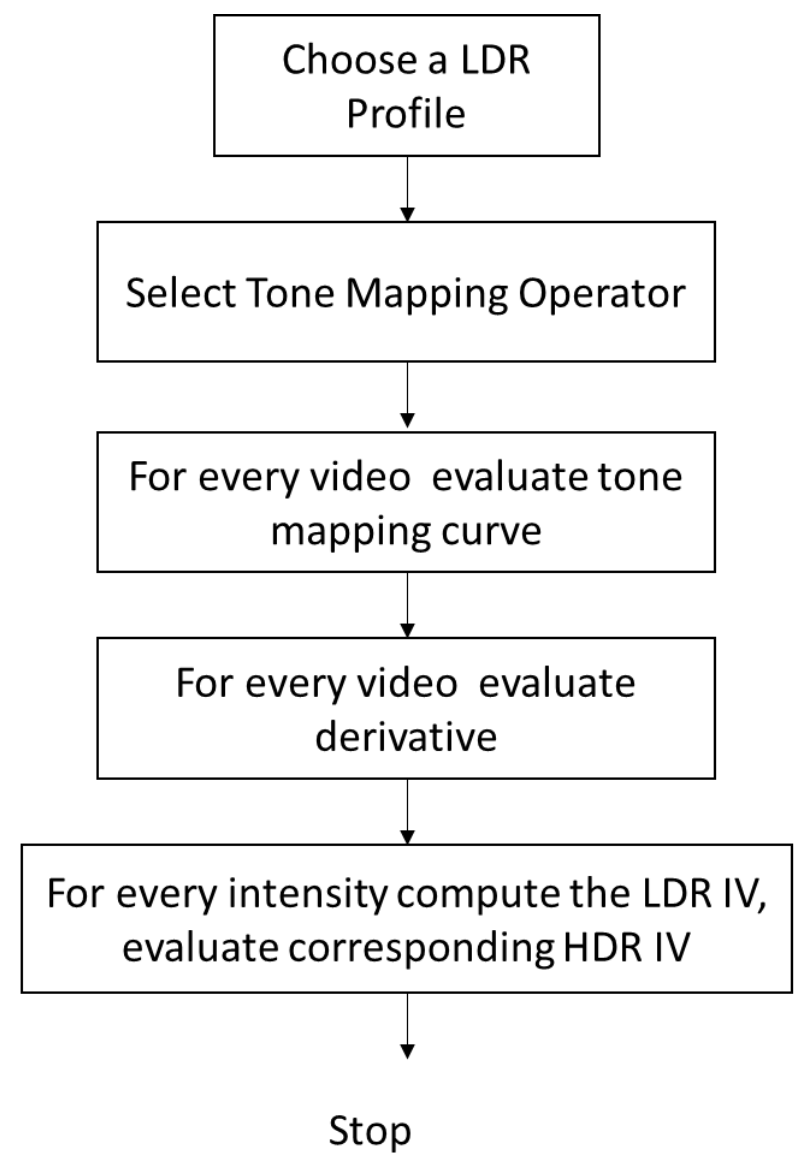

Figure.3 Proposed Flow Chart

Further the quantization is done acquiring the parameter of quantization for the intensity value, this parameter acquired in restricted using rounding. The determined parameters are more over conveyed to the decoder side thus limiting the time taken in computing the parameters for quantization. 
Journal of Innovative Image Processing (JIIP)

(2020)

Vol.02/ No. 01

Pages: 55-64

https://www.irojournals.com/iroiip/

DOI:_https://doi.org/10.36548/jiip.2020.1.006

The quantization performed varies for every block of the compressed video, and this requires the intensity of every block to be conveyed to the decoding side to overcome this the proposed model determines the total intensity utilizing the prediction of the current transform unit in the HEVC instead of using the intensity values. So a total intensity value (' $I V$ ') is determined for all the samples according to the equation 2 shown below.

$$
\operatorname{Avg}_{I V}=\frac{1}{X Y} \sum_{x=y=0}^{X Y} \text { predictor }(x, y)
$$

Where $\mathrm{X}$ and $\mathrm{Y}$ are the rows and columns respectively of the unit, so by this the actual intensity values are not conveyed to decoder, but enables the decoder to have a quantization relied on the intensity to be performed.

\section{Performance Analysis}

The proposed approach uses three real time sequence captured from the high definition cameras. The test material and the parameters involved in the assessment are listed in the tabulation. 1 below.

\begin{tabular}{|l|l|}
\hline \multicolumn{1}{|c|}{ Category } & \multicolumn{1}{c|}{ Model } \\
\hline Processor & Intel core i7-2600 \\
\hline Graphics & NVIDIA Ge FORCE GTX 650 Ti \\
\hline RAM & 4GB $133 \mathrm{MHz}$ \\
\hline HDD storage & $500 \mathrm{~GB}, 7200 \mathrm{RPM}$ \\
\hline SSD (Play back) & $\begin{array}{l}\text { 250 GB, Reading speed }=500 \mathrm{MB} / \mathrm{s} \\
\text { Writing Speed }=315 \mathrm{MB} / \mathrm{s}\end{array}$ \\
\hline $\begin{array}{l}\text { Video Play back } \\
\text { system }\end{array}$ & MATLAB \\
\hline
\end{tabular}

Table.1 Components Used

From the complete conclusion provided by the subjective assessment of the proposed model is listed below in the table. 2 on the basis of the delay and the bit rate reduction and the random access. For the three

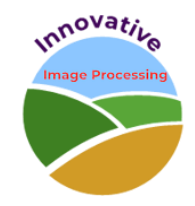


Journal of Innovative Image Processing (JIIP)

(2020)

Vol.02/ No. 01

Pages: 55-64

https://www.irojournals.com/iroiip/

DOI:_https://doi.org/10.36548/jiip.2020.1.006

sequence of input provided. The picture below in the figure. 4 is the input sequence fed and the table. 2 below shows the performance compared to the conventional methods.
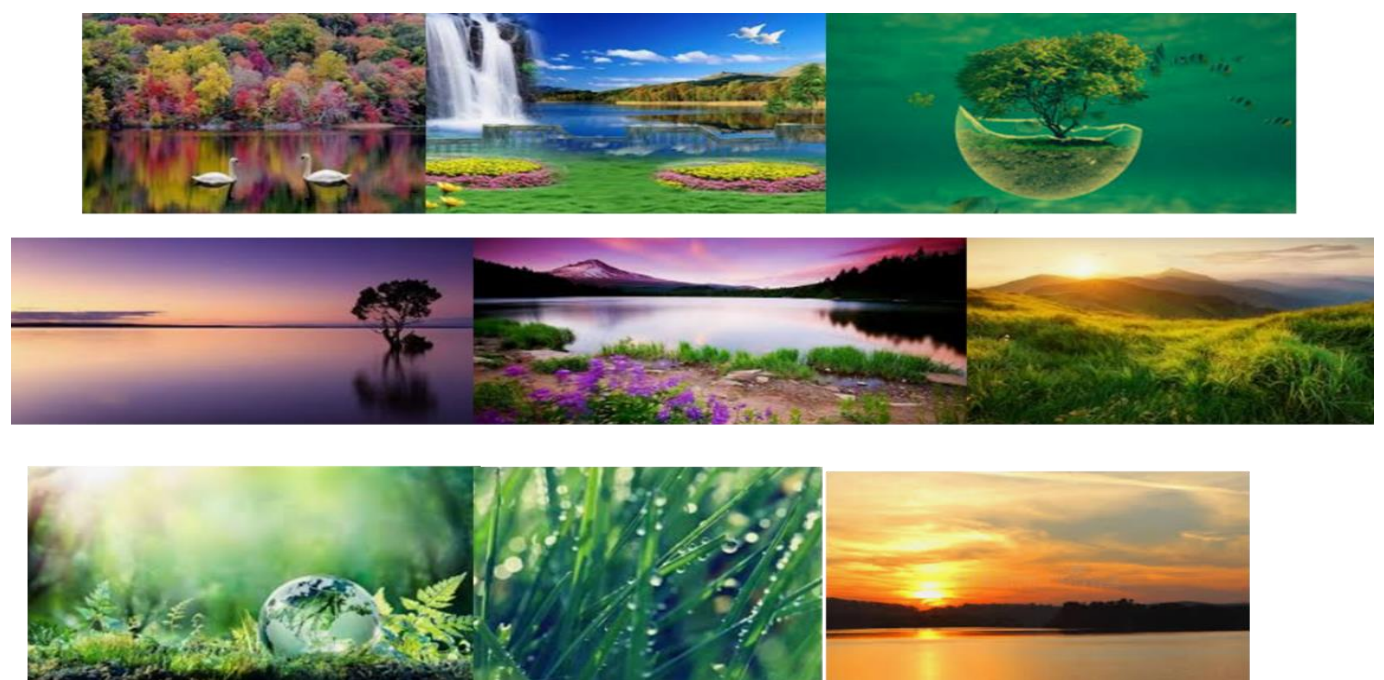

Figure. 4 Input Sequence

\begin{tabular}{|l|l|l|l|l|l|l|}
\hline \multirow{2}{*}{ Input } & \multicolumn{3}{|c|}{ Proposed } & \multicolumn{3}{c|}{ Conventional } \\
\cline { 2 - 7 } & Bitrate & Delay & $\begin{array}{c}\text { Random } \\
\text { Accesses }\end{array}$ & Bitrate & Delay & $\begin{array}{l}\text { Random } \\
\text { Accesses }\end{array}$ \\
\hline Input Sequence 1 & -6.69 & -08.91 & -10.70 & -0.54 & -1.44 & 1.88 \\
\hline Input Sequence 2 & -7.80 & -010.21 & -11.10 & -2.00 & -3.26 & 3.56 \\
\hline Input Sequence 3 & -5.79 & -04.31 & -8.81 & -0.38 & -0.56 & -0.59 \\
\hline
\end{tabular}

Table.2 Results Observed 
Journal of Innovative Image Processing (JIIP)

(2020)

Vol.02/ No. 01

Pages: 55-64

https://www.irojournals.com/iroiip/

DOI:_https://doi.org/10.36548/jiip.2020.1.006

\section{Conclusion}

The profile scaling using the tone mapping based on its distortion sensitivity and the perception based quantization is followed in the proposed method to compress the video preserving its quality. The performance assessment showed that the proposed method offers better compression with the reduced bit rate and delay and proper random access compared to the conventional SDR methods. The future work of the paper attempts to develop a strategy addressing the human eye sensitivity to the HDR contents chrominance and by utilizing the other phenomenon's of masking.

\section{References}

[1] Zhang, Yang, Dimitris Agrafiotis, and David R. Bull. "High dynamic range image \& video compression a review." In 2013 18th International Conference on Digital Signal Processing (DSP), pp. 1-7. IEEE, 2013.

[2] Korshunov, Pavel, and Touradj Ebrahimi. "A JPEG backward-compatible HDR image compression." In Applications of Digital Image Processing XXXV, vol. 8499, p. 84990J. International Society for Optics and Photonics, 2012.

[3] Miller, Scott, Mahdi Nezamabadi, and Scott Daly. "Perceptual signal coding for more efficient usage of bit codes." SMPTE Motion Imaging Journal 122, no. 4 (2013): 52-59.

[4] Zhang, Yang, Erik Reinhard, and David Bull. "Perception-based high dynamic range video compression with optimal bit-depth transformation." In 2011 18th IEEE international conference on image processing, pp. 1321-1324. IEEE, 2011.

[5] Wu, Hong Ren, and Kamisetty Ramamohan Rao, eds. Digital video image quality and perceptual coding. CRC press, 2017.

[6] Naccari, M., M. Mrak, D. Flynn, and A. Gabriellini. "Improving HEVC compression efficiency by intensity dependant spatial quantisation." In MPEG Meeting (Jul. 2012). 2012.

[7] Kumar, T. Senthil. "A Novel Method for HDR Video Encoding, Compression and Quality Evaluation." Journal of Innovative Image Processing (JIIP) 1, no. 02 (2019): 71-80.

[8] Manoharan, Samuel. "A smart image processing algorithm for text recognition, information extraction and vocalization for the visually challenged." Journal of Innovative Image Processing (JIIP) 1, no. 01 (2019): 31-38.

[9] Suma, V. "Computer Vision for Human-Machine Interaction-Review." Journal of trends in Computer Science and Smart technology (TCSST) 1, no. 02 (2019): 131-139.

[10] Koresh, M. H., and J. Deva. "Computer vision based traffic sign sensing for smart transport." J. Innovative Image Process.(JIIP) 1, no. 01 (2019): 11-19. 
Journal of Innovative Image Processing (JIIP)

(2020)

Vol.02/ No. 01

Pages: 55-64

https://www.irojournals.com/iroiip/

DOI:_https://doi.org/10.36548/jiip.2020.1.006

[11] Shakya, Subarna. "Virtual Restoration Of Damaged Archeological Artifacts Obtained From Expeditions Using 3d Visualization." Journal of Innovative Image Processing (JIIP) 1, no. 02 (2019): 102-110.

[12] Chandy, Abraham. "RGBD Analysis for Finding the Different Stages of Maturity of Fruits In Farming." Journal of Innovative Image Processing (JIIP) 1, no. 02 (2019): 111-121.

[13] Bindhu, V. "Biomedical Image Analysis Using Semantic Segmentation." Journal of Innovative Image Processing (JIIP) 1, no. 02 (2019): 91-101. 